\title{
Challenges that face the establishment of diabetes biobank in Jordan: a qualitative analysis of an online discussion forum
}

This article was published in the following Dove Press journal:

Journal of Multidisciplinary Healthcare

\author{
Omar F Khabour' \\ Ahmed Abu-Siniyeh ${ }^{1-3}$ \\ 'Department of Medical Laboratory \\ Sciences, Faculty of Applied Medical \\ Sciences, Jordan University of Science and \\ Technology, Irbid, Jordan; ${ }^{2}$ Department of \\ Medical Laboratory Sciences, Faculty of \\ Science, Al-Balqa' Applied University, As \\ Salt, Jordan; ${ }^{3}$ Clinical Laboratory Sciences \\ Department, College of Applied Medical \\ Sciences, Taif University, Taif, Kingdom of \\ Saudi Arabia
}

Introduction: Diabetes is common in Jordan with a prevalence of about $13 \%$ of the population. Establishment of a diabetes biobank in Jordan could have an enormous impact on the management and prevention of the disease.

Methods: In the current study, ethical challenges that might face the establishment of a biobank were examined by 28 researchers from the Middle East and North Africa region using an online discussion forum.

Results: All participants agreed on the importance of the establishment of a diabetes biobank in Jordan. The possible challenges that were discussed included confidentiality and privacy, informed consent, specimen ownership and participants' rights, data sharing, returning of research results and incidental findings, lack of legislations, and importance of social awareness and public engagements with biobanks.

Conclusion: In conclusion, participants support the establishment of a diabetes biobank in Jordan; however, some ethical issues should be considered to ensure the success of the biobank.

Keywords: diabetes, biobank, Jordan, MENA, ethics

\section{Introduction}

Biobanks are biorepository that store human samples for use in research. Since the start of biobanks during 1990s, biobanks have become an important bio-sample resource for biomedical research purposes, especially in proteomics, genomics, and personalized medicine. ${ }^{1}$ In addition, biobanks made a considerable contribution in the discovery of biomarkers, molecular diagnosis of diseases, translational medicine, and pathogenesis. ${ }^{2}$ The main duties of biobanks are collection, storage, and distribution of bio-samples including DNA/RNA, serum, urine, blood, and tissues. In addition, the patient's data such as demographics, disease history, and their associations with lifestyle, environmental factors, and comorbid health problems are also stored in biobanks and linked to the samples. ${ }^{3}$ Therefore, biobanks facilitate access to disease biospecimens and associated data.

Due to high demands for biobanks, different types aroused during the last years, including disease-specific, genetic, population-based, organ/tissue-specific, commercial, and virtual biobanks. ${ }^{4}$ Biobanks incorporate different types of studies, including epidemiological cohorts, clinical trials, and diagnostic and etiopathogenesis studies. ${ }^{2}$ While biobanks are important for the advances of human medical research, biobanks are usually confronted with several challenges, including financial, social, legal, and ethical issues.
Correspondence: Omar F Khabour Department of Medical Laboratory Sciences, Jordan University of Science and Technology, P.O. Box 3030, Irbid 22I I0, Jordan $\mathrm{Tel}+962720$ 1000-Ext 23852

Email khabour@just.edu.jo 
Among ethical issues are informed consent, privacy and confidentiality, results delivering to participants, public trust, commercialization, data exchange, sample ownership, and benefit sharing. ${ }^{5,6}$ In addition, different important aspects must be peered in mind before establishing a biobank such as adequate infrastructure, effective standardized record-keeping system, and quality management system under which the biobank will operate. $^{2}$

In Jordan, the prevalence of diabetes is currently close to $13 \%$ of the population, and it is increasing with time due to obesity, changes in dietary habits, and physical inactivity. ${ }^{7-9}$ Establishment of diabetes biobank in the country could significantly improve the quality of research that helps in both management and prevention of the disease. Jordan has an independent nonprofit organization called "The National Center for Diabetes, Endocrinology and Genetics" (NCDEG) which was established in 1996 and affiliated with the Higher Council for Science and Technology. The main objective of the center is to enhance diabetes research in Jordan that includes disease etiology and treatment. Establishing diabetes biobank in the center will expand the mission of the center and can open the door for Jordanian researchers to do targeted and high-quality research that target the disease in the country. In this study, ethical challenges that might face the establishment of diabetes biobank in Jordan were discussed among a group of researchers from Jordan and the Middle East and North Africa (MENA) region. The present findings highlighted some issues that should be considered for establishing a successful diabetes biobank in Jordan.

\section{Methods}

\section{Study design}

A question "Diabetes is common in Jordan. Establishment of diabetes biobank can support biomedical research in Jordan. Given the complex nature and ethical issues associated with biobanks, and the current research environment in Jordan: Do you support the establishment of such biobank in Jordan? What are the ethical challenges that might face such biobank in Jordan?" was asked to a group of researchers from Jordan and MENA through an online discussion forum. The forum was part of the activities of "Ethics of Genetic and Clinical studies" course that was offered by the Research Ethics Education Program in Jordan. All participants were fellows in the program. The forum was opened for 2 weeks, and 92 posts from a total of 28 participants were counted. The data of this discussion forum, which reflect the participant's opinions, were categorized into six ethical challenges: 1) privacy and confidentiality, 2) public awareness and trust, 3) informed consent, 4) financial and logistic support, 5) regulations, 6) sample ownership, and sharing. Systematic and consistent data collection during the discussions was guided by the predetermined theme' question. A professional researcher in qualitative research monitored the discussion. The monitoring process also includes promoting the participants to be engaged in the discussion by using some posts like "good point that needs more discussion" and "could you elaborate more" for validating the participants' opinions, which augmented the reliability of the collected data. ${ }^{10}$ To improve the objectivity of the study, a third researcher who was not participating in the discussion forum transcribed the collected data.

\section{Study population}

The online discussion forum is a part of the Responsible Conduct of Research (RCR) training program conducted online by San-Diego California University-USA, and funded by NIH to enhance the research ethical awareness among the Jordanian scientists. A total of 28 researchers were engaged with this discussion, including (18) Jordanian and (10) non-Jordanian from MENA region (Egypt, Algeria, Morocco, Yamen, Iraq, Sudan, Gaza, and Tunisia). Participant's positions were (4) research assistants (MSc degree) and (24) faculty members ( $\mathrm{PhD}$ degree) with different specialties including medicine, applied science, pharmacology, nursing, dentistry, and basic sciences. The Institutional Review Board at Jordan University of Science and Technology (IRB-JUST) approved the study. The committee applies the ethical standards as laid down in the 1964 Declaration of Helsinki and its later amendments. Written informed consent was taken from all participants as required by IRB-JUST.

\section{Results}

A group of researchers from Jordan and MENA region has participated in a discussion forum about the establishment of diabetes biobanks in Jordan and ethical challenges that might face such facility. All participants supported the establishment of diabetes biobank in Jordan and in the forum; the following challenges were discussed. 


\section{Challenges related to privacy and confidentiality}

Most of the researchers emphasized on the privacy and confidentiality of patient medical information as the leakage or abuse of such critical information may harm participants and decrease the trust between community and biobank.

A member (MM) said "The problem of participant' privacy and data confidentiality will keep being the major concern of biobanks in Jordan and the rest of the Arab world."

A female member (AS) mentioned "Building trust between people and the biobank is achieved by respecting participants' privacy and confidentiality,[...] Moreover, the samples and/or data might be abused by third parties such as insurance companies".

(AY) also added an opinion about this issue: "Maintaining a robust de-identification system that can protect the confidentiality of participants is essential in biobanks".

\section{Challenges related to public awareness and trust}

Community awareness was one of the important challenges that researchers pointed out. Varieties of measures were also proposed to improve awareness among the Jordanian population for the successful establishment of diabetes biobank.

A female member (HM) posted: "Public awareness regarding the basic elements of research (such as medical research, consent process, benefits/risks of participation) is important to enhance their active involvement in research in general and specifically in biobanks....... For increasing awareness, I think we can take advantage of Qatar experience with biobanks as they have formed a very informative website that contains all the information you need to know such as biobank importance, regulations, requirements, Islamic views...... We can also use social media networks as a new platform".

\section{Challenges related to the consent form}

The majority of participants raised this challenge, as the appropriate model of the consent form that should be adopted in biobanks is still a global debate.

(BA) posted: "Another important ethical issue is the type and preference of informed consent. Features of genetic studies and different elements related to biobank (stability of DNA over time, collection and storage of samples, the broad sharing of data) have challenged the traditional way of informed consent".
(LA) mentioned: "Also, new methods of consent will need to be created to replace the blanket consent to give subjects some real control over what they might consider inappropriate use of their information and biological material......"

\section{Challenges related to the financial and logistic support}

Some researchers raised challenges related to the financial and logistic support. Certainly, among important measures that must be considered before establishing any project are financial and logistical supports, because without resources and experiences, the project will fail.

One researcher stated: "Although that establishing a biobank for diabetes in Jordan is very challenging as it does need lots of financial and logistic supports as well as deep experience in doing such thing. I think that Jordan has the potential to establish such projects with the support of other countries who have stepped ahead in this area (USA, Europe... .etc.)."

While (WA) stated: "There is no doubt that establishing a diabetes biobank in Jordan will greatly enhance diabetes research if enough resources are available to perform this task. The resources range from financial support to personnel that have enough expertise in the field".

\section{Challenges related to regulations and laws}

Researchers highlighted the importance of having guidelines that regulate research activities in Jordan. Unfortunately, such guidelines are lacking in Jordan. This adds more challenges to the establishments of biobanks in the country.

A female member (HM) mentioned that: "New laws and rules must be issued to govern every aspect of an ethical research environment".

In more detailed answer (ZS), explained the need for research regulations in Jordan: "In Jordan, we need to create rules and laws to: protect the rights of donors, control the use of bio-samples and their storage, prevent the abuse of bio-samples, or the abuse of research results, handling issues related to disclosing research results or incidental findings......".

While another member stated about this issue "Jordan doesn't have formal legislation that governs the principles of development and utilization of biobanks with human samples". 


\section{Challenges related to the samples (handling, ownership, storage, and sharing)}

Researchers were wondering about handling, ownership, and storage of the donor samples as those are important issues that should be clearly noticed.

One participant (NM) was asking in her post: "Who will be responsible for keeping samples in biobanks and keeping information about these samples?".

(WK) was wondering: “...,who is the owner of the sample? Who has the right to decide how the sample should be used? How the results of the research disseminated? Would participants have the right to know results of research...?"

\section{Discussion}

A group of researchers from Jordan and MENA region using an online discussion forum discussed challenges that might face the establishment of diabetes biobank in Jordan. All participants agreed on the importance of establishing a diabetes biobank in the country. Among possible challenges that were raised by the researchers are confidentiality and privacy, informed consent, specimen ownership, data sharing, returning of research results to participants, lack of legislations, and importance of social awareness and public engagement in biobanks.

According to the results, all participants agreed on the need for a diabetes biobank in Jordan as the disease is common in Jordan and the region. Establishment of diabetes biobank will significantly improve the quality of diabetes research in the region. This includes investigation of diabetes risk factors, genetic predisposition, etiology of complications, and prognostic/predictive biomarkers. ${ }^{11}$ Among the expected benefits to the community are better prevention interventions/management of disease complications and improvement of quality of life and the patients' life expectancy. ${ }^{12}$

Privacy and confidentiality are one of the main ethical issues of diabetes biobank that researchers were concerned about. The researchers stressed that the patient's right to privacy should be protected, including their personal data, in order to maintain the trust between donors and biobank. Researchers also considered privacy and confidentiality as a general ethical challenge in the Arab world. Actually, despite cultural variances among different countries, privacy and confidentiality are still a universal concern that should be applied to ensure the successes of biobank projects. ${ }^{13,14}$ Different approaches were established in biobank system to resolve the problem of patient's privacy, including sample anonymization, de-identification and coding of samples, and data in a way that ensure privacy and confidentiality. ${ }^{15}$ Having laws and regulations and clear procedures to protect the participant's privacy and confidentiality are essential in enhancing public trust in research in general and in biobanks specifically.

Informed consent is considered, globally, one of the participant's right, as he/she should be aware about the nature of the biobank, the type of studies that will be conducted on the donated samples, and what are the benefits/risks of participation in biobanks. ${ }^{16-18}$ Participants stressed on the importance of improving the informed consent process to give subjects some control over what they might consider inappropriate use of their information and biological samples. In a previous study that investigated multiple sclerosis patients view regarding biobanks in Jordan, approximately $85 \%$ of the patients preferred open consent with $37 \%$ of them preferring to renew their consent. ${ }^{19}$ In a study that was conducted in South Africa, nearly half of the participants expressed a desire to be re-contacted for consent for future use of their samples. ${ }^{20}$ Different models of informed consent have been suggested and include open, broad, and blanket consent. ${ }^{21}$ The adopted model of informed consent in any future biobank should take into consideration public views, autonomy of patients, and concordance with research regulations in the country. ${ }^{17}$

A serious concern was shown by the researchers about applying the concept of ownership and benefit sharing of samples when establishing the biobank. Participants highlighted that in Jordan and most of the Arab world, we need regulations to protect the rights of donors, control the ownership and the use of bio-samples and generated data. This concern was highlighted in previous studies that examined the ethics of biobanks and commercial use of biobanks. ${ }^{22,23}$ This concern is of great importance as in Jordan there is no guideline related to ownership and control of human biological materials. The law concerning ownership and sharing of human biological material varies in different countries and in some countries such as the USA and UK, individuals do not retain ownership of the material donated for research. ${ }^{24}$ In general, researchers in the current study believe that legislations regarding ownership and sharing of samples are necessary for the success of the biobank project.

One challenge that was raised by the participants is the right of biobank participants to know the research findings including incidental findings. This issue is still of debate 
worldwide. Some people argue that usually participants donate their samples to researchers with no intention to know the results. ${ }^{25}$ In addition, some scientists believed that it is not a necessity to deliver the research results even though the participants ask for that. ${ }^{26}$ On the other hand, some studies showed the interests of the research participants in knowing the research results. For example, a qualitative study in the US reported such participants' desire in receiving their research results. ${ }^{27}$ In South Africa, most of the participants think when they donate their sample they have rights to receive research results. ${ }^{20}$ As a solution for this issue, it is recommended that returning of research findings including incidental ones be clarified to the participants during the informed consent process.

Among the challenges that were discussed by the participants were those related to the lack of public awareness and public involvement in biobanks. Participants considered public awareness of great importance to enhance active involvement of patients in biobanks. It is worth to mention that awareness about biobanks projects is increasing among Middle East populations. For example, a study involving hospital visitors, in Saudi Arabia, showed that around 60\% of people supported the biobanks idea and they are keen to get involved in such project. ${ }^{28}$ The public support of biobanks is also evident from a study that was conducted in Jordan and showed that $64 \%$ of participants were willing to participate in biobanks in case it is established in Jordan. ${ }^{19}$ Finally, strong public support was noticed when Qatar established the first biobank with wide-ranging data collection and storage of biosamples connected to health records and behavioral and physical characteristics of participants. ${ }^{29}$ This could be due to the transparency policy that was adopted in the project and included an informative website that explained biobank importance, regulations, requirements, and religion views. ${ }^{29}$ Some of the participants highlighted Qatar experience in biobanks and the importance of taking advantages from this case.

Finally, financial and logistic support is one of the difficult aspects that needs to be handled in establishing biobanks especially in a country like Jordan, which suffers from financial difficulties and the lack biobank experience. Financially speaking, the start-up investment for a biobank project needs a big fund for infrastructure, operating costs, sample acquisition, personnel, and other expenses, which are included into a model based on inadequate experience and limited data. ${ }^{30}$ It is worth to mention that Jordan has started to build its first cancer biobank (KHCCBIO), which is being established with support from four European organizations that covered all expertise and skills needed for biobank. ${ }^{31}$ This successful example can move forward the idea of establishing Diabetes biobank in Jordan. The Jordanian National Center for Diabetes, Endocrinology and Genetics is well established and has the infrastructure and the staff needed to handle and to manage the samples/generated data. Having a diabetes biobank in the center will expand its mission and can open the door for Jordanian researchers to do targeted and high-quality research that could eventually help in reducing the disease burden in the country.

Finally, it is worth to mention that the participant did not discuss some important challenges related to biobanks. This includes the need for trained staff/institution for the proper preservation of biological samples and the need for policies and strategies to invite and direct researchers. These aspects should be covered during the planning phase of the biobank project.

In conclusion, according to the views of involved researchers, the success of diabetes biobank in Jordan depends upon having clear legislations that regulate biobanks, public awareness and engagements and the protection of participant's rights, privacy, and confidentiality.

\section{Acknowledgments}

The Research Ethics Education Program in Jordan supported this study (US NIH grant number: 1R25TW010026-01). The authors would like to thank the Deanship of Research at Jordan University of Science and Technology for their support (2015/271).

\section{Disclosure}

The authors report no conflicts of interest in this work.

\section{References}

1. Greely HT. The uneasy ethical and legal underpinnings of large-scale genomic biobanks. Annu Rev Genom Hum Genet. 2007;8:343-364. doi:10.1146/annurev.genom.7.080505.115721

2. De Souza YG, Greenspan JS. Biobanking past, present and future: responsibilities and benefits. Aids. 2013;27(3):303-312. doi:10.1097/ QAD.0b013e32835c1244

3. Ryu E, Takahashi PY, Olson JE, et al. Quantifying the importance of disease burden on perceived general health and depressive symptoms in patients within the Mayo Clinic Biobank. Health Qual Life Outcomes. 2015;13:95. doi:10.1186/s12955-015-0285-6

4. Watson PH, Barnes RO. A proposed schema for classifying human research biobanks. Biopreserv Biobank. 2011;9(4):327-333. doi:10.1089/bio.2011.0020

5. Budimir D, Polasek O, Marusic A, et al. Ethical aspects of human biobanks: a systematic review. Croat Med J. 2011;52(3):262-279.

6. Hansson MG. Ethics and biobanks. Br J Cancer. 2009;100(1):8-12. doi:10.1038/sj.bjc. 6604795 
7. Alghadir A, Alghwiri AA, Awad H, Anwer S. Ten-year diabetes risk forecast in the capital of Jordan: Arab diabetes risk assessment questionnaire perspective-a strobe-complaint article. Medicine. 2016;95(12):e3181. doi:10.1097/MD.0000000000004864

8. Al-Nsour M, Zindah M, Belbeisi A, Hadaddin R, Brown DW, Walke $\mathrm{H}$. Prevalence of selected chronic, noncommunicable disease risk factors in Jordan: results of the 2007 Jordan behavioral risk factor surveillance survey. Prev Chronic Dis. 2012;9:E25.

9. Zindah M, Belbeisi A, Walke H, Mokdad AH. Obesity and diabetes in Jordan: findings from the behavioral risk factor surveillance system, 2004. Prev Chronic Dis. 2008;5(1):A17.

10. Tobin GA, Begley CM. Methodological rigour within a qualitative framework. $J$ Adv Nurs. 2004;48(4):388-396. doi:10.1111/j.13652648.2004.03207.x

11. Petersen ER, Nielsen AA, Christensen H, et al. Vejle Diabetes Biobank a resource for studies of the etiologies of diabetes and its comorbidities. Clin Epidemiol. 2016;8:393-413. doi:10.2147/CLEP.S113419

12. Van't Riet E, Schram MT, Abbink EJ, et al. The diabetes pearl: diabetes biobanking in The Netherlands. BMC Public Health. 2012;12:949. doi:10.1186/1471-2458-12-949

13. Kaufman DJ, Murphy-Bollinger J, Scott J, Hudson KL. Public opinion about the importance of privacy in biobank research. Am J Hum Genet. 2009;85(5):643-654. doi:10.1016/j.ajhg.2009.10.002

14. Lemke AA, Wolf WA, Hebert-Beirne J, Smith ME. Public and biobank participant attitudes toward genetic research participation and data sharing. Public Health Genom. 2010;13(6):368-377. doi:10.1159/000276767

15. Knoppers BM, Zawati MH, Kirby ES. Sampling populations of humans across the world: ELSI issues. Annu Rev Genomics Hum Genet. 2012;13:395-413. doi:10.1146/annurev-genom-090711-163834

16. Eriksson S, Helgesson G. Potential harms, anonymization, and the right to withdraw consent to biobank research. Eur J Hum Genet. 2005;13(9):1071-1076. doi:10.1038/sj.ejhg.5201458

17. Hallinan D, Friedewald M. Open consent, biobanking and data protection law: can open consent be 'informed' under the forthcoming data protection regulation? Life Sci Soc Policy. 2015;11:1. doi:10.1186/s40504-014-0020-9

18. Hoeyer K, Olofsson BO, Mjorndal T, Lynoe N. The ethics of research using biobanks: reason to question the importance attributed to informed consent. Arch Intern Med. 2005;165(1):97-100. doi:10.1001/archinte.165.1.97

19. Ahram M, Othman A, Shahrouri M. Public support and consent preference for biomedical research and biobanking in Jordan. Eur J Hum Genet. 2013;21(5):567-570. doi:10.1038/ejhg.2012.213
20. Moodley K, Sibanda N, February K, Rossouw T. "It's my blood": ethical complexities in the use, storage and export of biological samples: perspectives from South African research participants. BMC Med Ethics. 2014;15:4. doi:10.1186/1472-6939-15-4

21. Beskow LM, Lin L, Dombeck CB, Gao E, Weinfurt KP. Improving biobank consent comprehension: a national randomized survey to assess the effect of a simplified form and review/retest intervention. Gene Med. 2017;19(5):505-512. doi:10.1038/gim.2016.157

22. Bledsoe MJ. Ethical legal and social issues of biobanking: past, present, and future. Biopreserv Biobank. 2017;15(2):142-147. doi:10.1089/bio.2017.0030

23. Joly Y, Dalpe G, So D, Birko S. Fair shares and sharing fairly: a survey of public views on open science, informed consent and participatory research in biobanking. PLoS One. 2015;10(7): e0129893. doi:10.1371/journal.pone.0129893

24. Caulfield T, Burningham S, Joly Y, et al. A review of the key issues associated with the commercialization of biobanks. J Law Biosci. 2014;1(1):94-110. doi:10.1093/jlb/lst004

25. Forsberg JS, Hansson MG, Eriksson S. Changing perspectives in biobank research: from individual rights to concerns about public health regarding the return of results. Eur J Hum Genet. 2009;17 (12):1544-1549. doi:10.1038/ejhg.2009.87

26. Meulenkamp TM, Gevers SK, Bovenberg JA, Koppelman GH, van Hylckama Vlieg A, Smets EM. Communication of biobanks' research results: what do (potential) participants want? Am J Med Genet. 2010;152A(10):2482-2492. doi:10.1002/ajmg.a.33617

27. Murphy J, Scott J, Kaufman D, Geller G, LeRoy L, Hudson K. Public expectations for return of results from large-cohort genetic research. Am J Bbioethics. 2008;8(11):36-43. doi:10.1080/ 15265160802513093

28. Al-Jumah MA, Abolfotouh MA. Public perception and attitude of saudis toward organ and tissue donation. Biopreserv Biobank. 2011;9 (1):21-27. doi:10.1089/bio.2010.0025

29. Al Kuwari H, Al Thani A, Al Marri A, et al. The Qatar Biobank: background and methods. BMC Public Health. 2015;15:1208. doi:10.1186/s12889-015-2522-7

30. Macleod AK, Liewald DC, McGilchrist MM, Morris AD, Kerr SM, Porteous DJ. Some principles and practices of genetic biobanking studies. Eur Respir J. 2009;33(2):419-425. doi:10.1183/ 09031936.00043508

31. Barr MP, Souan L, MacGabhann P, et al. The establishment of an ISO compliant cancer biobank for Jordan and its neighboring countries through knowledge transfer and training. Biopreserv Biobank. 2014;12(1):3-12. doi:10.1089/bio.2013.0072

\section{Publish your work in this journal}

The Journal of Multidisciplinary Healthcare is an international, peerreviewed open-access journal that aims to represent and publish research in healthcare areas delivered by practitioners of different disciplines. This includes studies and reviews conducted by multidisciplinary teams as well as research which evaluates the results or conduct of such teams or healthcare processes in general. The journal covers a very wide range of areas and welcomes submissions from practitioners at all levels, from all over the world. The manuscript management system is completely online and includes a very quick and fair peer-review system. Visit http://www.dovepress.com/testimonials. php to read real quotes from published authors. 